\title{
The Detection Method of Cell-Derived Exosomes
}

\author{
Jianxiong $\mathrm{Hu}^{1}$, Kangying Wang ${ }^{4}$, Chengfei Zhao ${ }^{2,3 *}$, Qihong Cai ${ }^{2,3 *}$ and Linghong Pan ${ }^{2,3}$ \\ ${ }^{1}$ Intensive Care Unit (ICU), The Affiliated Hospital of Putian University, China \\ ${ }^{2}$ Department of Pharmacy, Putian University, China \\ ${ }^{3}$ Higher Educational Key Laboratory for Pharmaceutical Analysis and Laboratory Medicine of Fujian province, Putian University, China \\ ${ }^{4}$ Department of Clinical Laboratory, The Affiliated Hospital of Putian University, China
}

Submission: December 18, 2017; Published: February 02, 2018

*Corresponding author: Chengfei Zhao, Department of Pharmacy, Pharmacy and Medical Technology School, Putian University, 1133 Xueyuan Road, Chengxiang District, Putian 351100, PR China; Email: zhaochengfei209@163.com

Qihong Cai, Department of Pharmacy, Pharmacy and Medical Technology School, Putian University, 1133 Xueyuan Road, Chengxiang District, Putian 351100, PR China; Email: cqh118@163.com

\begin{abstract}
Exosomes are small extracellular vesicles $(30-100 \mathrm{~nm})$ from various cells, and have emerged as a promising biomarker of some diseases. Quantitative detection of cell-derived exosome is appealing and challenging. In recent years, many kinds of methods have emerged for the detection and analysis of exosomes, including electrochemical method, microfluidic technology, microarray chip and colorimetric method. We summarize the current detection methods of exosomes and highlight the development direction of detection method for exosomes.

Keywords: Exosomes; Electrochemical biosensors; Microarray chip; Microfluidic platform; Flow immunoassay; Colorimetric method
\end{abstract}

\section{Introduction}

Exosomes were initially reported in 1983 by Johnstone and colleagues while culturing reticulocytes [1]. It is already very clear that exosomes are cell-derived lipid bilayer vesicles that range from 30 to $100 \mathrm{~nm}$ in diameter [2], are released by many types of cells, such as immune cells and tumor cells [3]. And Exosomes can be found in most bodily fluid, including blood, urine, saliva, amniotic fluid, breast milk, hydrothoracic fluid, and ascitic fluid, as well as in culture medium of most cell types [4]. Exosomes are also an extracellular subset containing microRNAs, messenger RNAs (mRNA), DNA fragments, lipids and proteins for cell communication [5], are transported through the bloodstream and other body fluids [6]. A series of emerging evidence have suggested that exosomes may play an important role in the interaction between tumor cells and their surrounding cells in the tumor microenvironment. However, the detection of exosomes remains challenging and restricts its clinical application. According to the existing literatures, the review summarizes and analyzes the latest detection methods of exosomes, and prospects the detection technology of the exosomes.

\section{Electrochemical Biosensor}

Electrochemical biosensors combine the sensitivity of electroanalytical methods with the inherent bioselectivity of the biological component [7]. Wang et al. [8] developed a nanotetrahedron (NTH)-assisted electrochemical aptasensor for direct capture and detection of hepatocellular exosomes, and provided a proof-of-concept for sensitive and efficient quantification of tumor-derived exosomes [8]. The NTH-assisted electrochemical aptasensor combined the strengths of advanced aptamer technology, DNA-based nanostructure and portable electrochemical devices. Aptamer LZH8 with superior binding selectivity on target hepatocarcinoma cells (HepG2) was used for the proposed electrochemical aptasensor. Yadav et al. [9] reported an electrochemical method to directly quantify the breast cancer cell-derived exosomes in cell culture media [9]. The method exhibited an excellent specificity for human epidermal growth factor receptor 2 (+) BT474 cell-derived exosomes (detection limit of $4.7 \times 10^{5}$ exosomes $/ \mu \mathrm{L}$ ) with an RSD of $<4.9 \%$ $(n=3)$. Zhou et al. [10] developed an electrochemical biosensor based on aptamers specific to exosome transmembrane protein 
CD63 for the quantitative detection of exosomes [10]. The biosensors functioned well both in "clean" (HEPES Buffer) and "dirty" (DMEM cell culture media containing 10\% fetal bovine serum) solutions with a detection limit of $1 \times 106$ particles $/ \mathrm{mL}$ and a linear range extending two orders of magnitude to $1 \times 108$ particles $/ \mathrm{mL}$. These three studies only detected exosomes in cell culture media, and didn't involve the detection of exosomes in body fluid samples.

\section{Microarray Chip}

Microarrays with biomolecules, cells and tissues immobilized on solid substrates are important tools for biological research, including genomics, proteomics, and cell [11]. Ibsen et al. [12] developed an alternating current electrokinetic (ACE) microarray chip device to rapidly isolate and recover glioblastoma exosomes from undiluted human plasma samples [12]. The detection of the exosome specific pattern of external CD63 membrane protein and internal TSG101 protein 17 confirms the presence of glioblastoma exosomes among the collected extracellular vesicles by the ACE microarray chip device. The ACE device achieved the direct detection of exosomes in human plasma. Daaboul et al. [15] presented a method based on Single Particle Interferometric Reflectance Imaging Sensor (SP-IRIS) that allowed multiplexed phenotyping and digital counting of various populations of individual exosomes $(>50 \mathrm{~nm})$ captured on a microarray-based solid phase chip [13]. The SP-IRIS for the detection of exosomes purified from HEK cell line showed a good correlation for both capture antibodies CD63 and CD81, and detection limits of $5.07 \times 10^{9}$ particles $/ \mathrm{mL}$ for CD63 antibody and $3.94 \times 109$ particles/mL for CD81 antibody. The imaging method could capture exosomes from a very small volume $(20 \mathrm{uL})$ of human cerebrospinal fluid by using antibodies directed against tetraspanins.

\section{Microfluidic Platform}

Microfluidic systems can be designed to obtain and process measurements from small volumes of complex fluids with efficiency and speed [14]. Zhao et al. [15] employed a microfluidic ExoSearch chip for diagnosis of ovarian cancer by multiplexed measurement of three exosomal tumor markers (CA-125, EpCAM, CD24) using a training set of plasmas from ovarian cancer patients [15]. The ExoSearch chip combined on-chip continuous-flow mixing and immunomagnetic isolation with an in situ, multiplexed exosome immunoassay, and was applied for ovarian cancer diagnosis via quantifying a panel of tumor markers from exosomes in $20 \mu \mathrm{L}$ of blood plasma within $40 \mathrm{~min}$. Zhang et al. [16] developed a microfluidic platform based on a new graphene oxide/polydopamine (GO/PDA) nano-interface for the analysis of circulating exosomes [16]. CD81 mAb served for the capture antibody, and a cocktail of biotinylated mAbs of CD9 and CD81 and EpCAM acted as the detection antibody in the study. The platform was applied to discriminate ovarian cancer patients from healthy controls by the quantitative detection of circulating exosomes directly from $2 \mu \mathrm{L}$ of plasma without sample processing. These two studies provided a feasible microfluidic platform to directly detect exosomes in blood plasma from patients and to facilitate basic investigation of exosomes.

\section{Flow Immunoassay}

Lateral flow immunoassay represents a well established and appropriate technology among rapid assays because of its low cost and user-friendliness [17]. Oliveira-Rodríguez et al. developed a novel lateral flow immunoassay (LFIA) for the detection of exosomes based on the use of tetraspanins as targets [18]. The platform was used to detect exosomes purified from different sources, including cell culture supernatants, human plasma and urine, and completed an one-step assay of exosomes in $15 \mathrm{~min}$ with detection limit of $8.54 \times 10^{5}$ exosomes/ $\mu \mathrm{L}$. And the platform selected a blend of anti-CD9 and anti-CD81 as capture antibodies and anti-CD63 labeled with gold nanoparticles as detection antibody. The work achieved a rapid quantification of exosome from different biological samples and provided promising techniques for clinical diagnosis.

\section{Colorimetric Method}

Colorimetry is that a well-designed chemical interaction between the analyte and NPs surroundings leads to a change of color allowing the visual detection of the target analyte [19]. Yao et al. [20] reported a visible and colorimetric aptasensor based on DNA-capped single-walled carbon nanotubes for the detection of exosomes, and estimated the linear range from $1.84 \times 106$ to $2.21 \times 107$ particles $/ \mu \mathrm{L}$ with detection limit of $5.2 \times 10^{5}$ particles/ $\mu \mathrm{L}[20]$. An aptamer specific to exosomes transmembrane protein CD63 was used for the proposed colorimetric aptasensor. The aptasensor was also used to quantify exosomes in serum from breast cancer patients and healthy individuals.

\section{Conclusion and Outlook}

The importance of exosomes is self-evident for cancer research. At present, many methods, including electrochemical biosensor, microarray chip, microfluidic platform, flow immunoassay and colorimetric method, provide the detection technology of exosomes. Electrochemical methods afford a sensitive and rapid analysis strategy, but cannot directly detect exosomes in the biological sample. Colorimetric method possesses a simple visual advantage, but is helpless for the direct detection of exosomes in serum. Lateral flow immunoassay is homogeneous and heterogeneous analytical methods which enables the separation of reacted products from unreacted products without any additional precipitation or washing procedure, and provide a promising platform for detecting exosomes. Microarray chip can afford many molecular signatures for cells, tissues and disease states that can be used for disease diagnosis, prediction, prevention and drug discovery, and is more suitable for the analysis and detection of exosomes in complex samples. Microfluidic chip have had a considerable impact on the fields of biomedical diagnostics, and is better to analyze the exosomes in different body fluid. According to the above analysis 
and summary, the studies of exosomes need to combine various advanced techniques to obtain more comprehensive information of exosomes and to achieve the diagnosis of related diseases in clinical.

\section{Acknowledgement}

We gratefully acknowledge the financial support of the Natural Science Foundation of Fujian Province (2017J01547 and 2017J01346), the Key Projects of Science and Technology Plan of Fujian Province (2015Y0030), the Scientific Research Project of Fujian Provincial Colleges and Universities (JK2014045), and the Science and Technology Plan Projects of Putian City (2013S01(2)).

\section{Conflict of Interest}

All authors declare no conflict of interest.

\section{References}

1. Pan BT, Johnstone RM (1983) Fate of the transferrin receptor during maturation of sheep reticulocytes in vitro: selective externalization of the receptor. Cell 33(3): 967-978.

2. Lin LY, Du LM, Cao K, Huang Y, Yu PF, et al. (2016) Tumour cell-derived exosomes endow mesenchymal stromal cells with tumour-promotion capabilities. Oncogene 35(46): 6038-6042.

3. Zhang X, Pei Z, Chen J, Ji C, Xu J, et al. (2016) Exosomes for immunoregulation and therapeutic intervention in cancer. J Cancer $7(9):$ 1081-1087.

4. Lin J, Li J, Huang B, Liu J, Chen X, et al. (2015) Exosomes: novel biomarkers for clinical diagnosis. Sci World J, 2015.

5. Kahlert C, Kalluri R (2013) Exosomes in tumor microenvironment influence cancer progression and metastasis. J Mol Med 91(4): 431437.

6. Yellon DM, Davidson SM (2014) Exosomes:nanoparticles involved in cardioprotection? Circ Res 114(2): 325-332.

7. Ronkainen NJ, Halsall HB, Heineman WR (2010) Electrochemical biosensors. Chem Soc Rev 39(5): 1747-1763.
8. Wang S, Zhang L, Wan S, Cansiz S, Cui C, et al. (2017) Aptasensor with expanded nucleotide using DNA nanotetrahedra for electrochemical detection of cancerous exosomes. ACS Nano 11(4): 3943-3949.

9. Yadav S, Boriachek K, Islam MN, Lobb R, Möller A, et al. (2017) An Electrochemical Method for the Detection of Disease-Specific Exosomes. ChemElectroChem 4(4): 967-971.

10. Zhou Q, Rahimian A, Son K, Shin DS, Patel T, et al. (2016) Development of an aptasensor for electrochemical detection of exosomes. Methods 97: 88-93.

11. Barbulovic-Nad I, Lucente M, Sun Y, Zhang M, Wheeler AR, et al. (2006) Bio-microarray fabrication techniques-a review. Crit Rev Biotechnol 26(4): 237-259.

12. Ibsen SD, Wright J, Lewis JM, Kim S, Ko SY, et al. (2017) Rapid isolation and detection of exosomes and associated biomarkers from plasma. ACS Nano 11(7): 6641-6651.

13. Daaboul GG, Gagni P, Benussi L, Bettotti P, Ciani M, et al. (2016) Digital detection of exosomes by interferometric imaging. Sci Rep-UK 6.

14. Yager P, Edwards T, Fu E, Helton K, Nelson K, et al. (2006) Microfluidic diagnostic technologies for global public health. Nature 442(7101): 412-418.

15. Zhao Z, Yang Y, Zeng Y, He M (2016) A microfluidic ExoSearch chip for multiplexed exosome detection towards blood-based ovarian cancer diagnosis. Lab Chip 16(3): 489-496.

16. Zhang P, He M, Zeng Y (2016) Ultrasensitive microfluidic analysis of circulating exosomes using a nanostructured graphene oxide/ polydopamine coating. Lab Chip 16(16): 3033-3042.

17. Chen A, Yang S (2015) Replacing antibodies with aptamers in lateral flow immunoassay. Biosens Bioelectron 71: 230-242.

18. Oliveira-Rodríguez M, López-Cobo S, Reyburn H T, et al. (2016) Development of a rapid lateral flow immunoassay test for detection of exosomes previously enriched from cell culture medium and body fluids. J Extracell Vesicles 5(1): 31803.

19. Vilela D, González MC, Escarpa A (2012) Sensing colorimetric approaches based on gold and silver nanoparticles aggregation: Chemical creativity behind the assay. A review. Anal Chim Acta 751: 24-43.

20. Xia Y, Liu M, Wang L, Yan A, He W, et al. (2017) A visible and colorimetric aptasensor based on DNA-capped single-walled carbon nanotubes for detection of exosomes. Biosens Bioelectron 92: 8-15.

\section{Your next submission with Juniper Publishers} will reach you the below assets

- Quality Editorial service

- Swift Peer Review

- Reprints availability

- E-prints Service

- Manuscript Podcast for convenient understanding

- Global attainment for your research

- Manuscript accessibility in different formats

( Pdf, E-pub, Full Text, Audio)

- Unceasing customer service

Track the below URL for one-step submission https://juniperpublishers.com/online-submission.php 